\title{
Smoking and Dental Stain among Fifth Grade Dental Students, University of Baghdad
}

\author{
Dina Hamid Obaid ${ }^{1}$, Noor Hayder Fadhil ${ }^{2}$ \\ ${ }^{1}$ Department of Orthodontics, College of Dentistry, University of Baghdad, Iraq \\ ${ }^{2}$ Department of Conservative Dentistry, College of Dentistry, University of Baghdad, Iraq
}

\begin{abstract}
Background: Smoking is the largest preventable risk factor for morbidity and mortality in developed countries. Smoking has many effects on the body like lung cancer, oral cancer, heart disease, and strokes typically do not occur until years. Smoking also has many effects on oral cavity; one of these effects is teeth staining or called (smoking stain). Aims of the study: To compare occurrence of dental stain between smokers and nonsmokers, among dental students, and to explore the relation between duration of, number of cigarettes smoking and the type of dental stain. Materials and Methods: The sample collected was 40 males (23 years old), $5^{\text {th }}$ grade dental student's university of Baghdad, divided into two groups 20 smokers and 20 control. All teeth of the students were examined for the presence of dental stain, distribution of that stain and the color of the stain according to Leung (1950). Results: The percentage of dental stain was $90 \%$ among smokers, while it is $65 \%$ among non-smokers. The brown dental stain was the comments type among both groups, followed by the yellow stain, while none was seen with the green and orange stain. Results demonstrated that the duration of smoking and the numbers of cigarettes smoked did not affect dental stain. Conclusion: Smoking may have an influence on extrinsic dental stain.
\end{abstract}

Keywords: Smoking, dental stain, leung index

\section{Introduction}

Smoking is the largest preventable risk factor for morbidity and mortality in developed countries. A number of clinical studies and regional health surveys have found an association between smoking and poor oral health [1].

Cigarette smoke contains over 4000 chemical compounds. More than 60 of these have been identified as cancer causing [2] like lung cancer, oral cancer(lip and tongue), pharynx cancer, esophagus cancer, pancreas cancer, urinary bladder cancer, nasal cavity cancer, sinuses cancer, stomach cancer, liver cancer, kidney cancer, uterine cervix cancer and leukemia [3]. For example of these compounds are carbon monoxide, found in car exhausts, binds to red blood cells and reduces the ability of blood to carry oxygen. Hydrogen cyanide kills the cilia (small hair cells) that clear particles from the lungs (including smoke particles for smokers) [2] and nicotine that has direct effect on the brain due to its addiction power[4].

Many teenagers and adults think that there are no effects of smoking on their bodies until they reach middle age. In addition to cancers smoking also causes heart disease, and stroke typically do not occur until years after a person's first cigarette. However, there are many serious harms from smoking that occur much sooner. In fact, smoking has numerous immediate health effects on the brain and on the respiratory, cardiovascular, gastrointestinal, immune and metabolic systems. While these immediate effects do not all produce noticeable symptoms, most begin to damage the body with the first cigarette, sometimes irreversibly and rapidly produce serious medical conditions and health consequences. In addition to the general health risks posed by smoking, it also adversely affects oral health [5].

Tobacco use in any form cigarette, cigar, pipe and smokeless (spit) tobacco increases the risk for a variety of oral health Conditions, including:
- Periodontal disease

- Tooth decay, bad breath and stained teeth

- Partial and complete tooth loss

- Oral cancer

- Candida Albicans.

- Smoker's Melanosis

- Nicotinic stomatitis

- Black Hairy Tongue [6].

One of smoking effects on oral health is teeth staining or called \{smoking stain $\}$ [5].

Dental stain is the tooth discoloration that either intrinsic (occur following change to the structural composition or thickness of the tooth) or extrinsic (occur on the external tooth surface) [7]. It is widely recognized that today's youth and appearance oriented culture prizes an attractive smile and white teeth. Dental stains differ in etiology, Appearance, composition, location, severity and degree of adherence. Attraction of material to the tooth surface plays a critical role in the deposition of extrinsic dental stains. However the mechanism that determines the adhesion strength is not completely understood. Discolorations of the teeth and restorations occur in three general ways: (1) stain adheres directly to the surfaces, (2) stain contained within calculus and soft deposits and (3) stain incorporated within the tooth structure or the restorative material- Instructional and clinical procedures apply to all three. The first two types may be removed by scaling or polishing. Certain stains may be prevented by the patient's routine personal care. Thick deposits of stain can provide a rough surface on which dental biofilm can collect and irritate die adjacent gingiva. Certain stains provide a means of evaluating oral cleanliness and the patients' habits of personal care [8].

The extrinsic stain is either metallic or nonmetallic and the colors are brown, black, orange, green, yellow, violet or redblack stain [7]. 


\section{International Journal of Science and Research (IJSR) \\ ISSN (Online): 2319-7064}

Index Copernicus Value (2016): 79.57 | Impact Factor (2015): 6.391

While the intrinsic stain has a number of metabolic diseases and systemic factors are known to affect the developing dentition and cause discoloration as a consequence like;

1. Alkaptonuria.

2. Congenital erythropoietic porphyria.

3. Congenital hyperbilirubinaemia.

4. Amelogenesis imperfect.

5. Dentinogenesis imperfect.

6. Tetracycline staining.

7. Fluorosis.

8. Enamel hypoplasia.

9. Pulpal haemorrhagic products. [7].

According to knowledge available, no previous Iraqi study was able to be found regarding smoking and dental stain, for this reason, this study was designed.

\section{Materials and Methods}

2.1 The subjects: The subjects were 40 males (23 years old), $5^{\text {th }}$ grade dental students university of Baghdad.

\subsection{Design of the study}

All the subjects were informed about the purposes of the Investigation and consented to its protocol. All teeth of the subjects were examined for the presence of dental stain, distribution of that stain, and the color of the stain. In addition to information taken from the subjects regarding number of cigarettes smoked per day, and number of years the patient had smoke. The study subjects divided into two groups:

- Control group (nonsmoker): Twenty males didn’t present any history of smoking.

- Smoker group: Twenty male smokers with different durations and dose.

The examination done by the use of disposable mirrors.

\subsection{Method of examination:}

The index used in this study is Leung (1950) for stain examination:

$0=$ no color present

$1=$ black color

$2=$ brown color

$3=$ yellow color

$4=$ green color

$5=$ orange color

The design of index we used permitted scoring the presence of more than one color in each segment in the same subject.

\section{Results}

\subsection{The subjects:}

The sample collected was 40 males (23years old), $5^{\text {th }}$ grade dental students university of Baghdad, It consisted of 20 smokers and 20 control group.

Figure (3.1) shows the percentage of dental stain among smokers and non-smokers. That the percentage of dental stain is $90 \%$ among smokers, while it is $65 \%$ among nonsmokers.

Figure (3.2) illustrate the percentage of each type of dental stain among both smokers and non-smokers, it shows that the percentage of brown stain was 50\% among smokers and $55 \%$ among non-smokers.

The percentage of black stain was $15 \%$ among smokers and $5 \%$ among non-smokers, while the percentage of yellow stain was $35 \%$ among smokers and $20 \%$ among nonsmokers.

None of the subjects whose teeth have green or orange stain where found in this study.

Table (3-1) shows the distribution of smokers with stain according to the duration of smoking.

It shows that the numbers of smokers when the duration was 1-4 years are 6 smokers, 3 subjects had brown stain 4 subjects had yellow stain and no black stain present.

When the duration was 5-8 years, the smokers were 8 subjects one of them has no stain while 2 subjects had black stain 3 subjects had brown stain and 3 subjects had yellow stain.

Finally when the duration was 9-12 years, the smokers were 6 subjects one of them without stain and one had black stain while 4 subjects had brown stain and no one has yellow stain.

Whatever was the duration, none of subjects had orange or green stain.

Table (3-2) shows the relation between number of cigarette smoking and type of dental stain, it shows that only two subjects had no stain when the number is from 1cigarette to 40 cigarettes, while 3 subjects had black stain, 9 subjects had brown stain and 7 subjects had yellow stain. While when the number is from 41-80 we find only one brown stain.

None of the subjects whose teeth have green or orange stain where found in this study.

\subsection{Data analysis:}

Data processing and analysis were carried out by estimation of the percentage only. 


\section{International Journal of Science and Research (IJSR) \\ ISSN (Online): 2319-7064 \\ Index Copernicus Value (2016): 79.57 | Impact Factor (2015): 6.391}

Table 1: Distribution of smokers with stain according to duration of smoking

\begin{tabular}{|c|c|c|c|c|c|c|c|}
\hline $\begin{array}{c}\text { Duration } \\
\text { In years }\end{array}$ & $\begin{array}{c}\text { Smoker } \\
\text { number }\end{array}$ & $\begin{array}{c}\text { NO } \\
\text { stain }\end{array}$ & Black & Brown & Yellow & Green & Orange \\
\hline $1-4$ & 6 & 0 & 0 & 3 & 4 & 0 & 0 \\
\hline $5-8$ & 8 & 1 & 2 & 3 & 3 & 0 & 0 \\
\hline $9-12$ & 6 & 1 & 1 & 4 & 0 & 0 & 0 \\
\hline
\end{tabular}

Table 2: Types of stain according to number of cigarettes

\begin{tabular}{|c|c|c|c|c|c|c|}
\hline No. of cigarettes & No stain & Black & Brown & Yellow & Green & Orange \\
\hline $1-40$ & 2 & 3 & 9 & 7 & 0 & 0 \\
\hline $41-80$ & 0 & 0 & 1 & 0 & 0 & 0 \\
\hline
\end{tabular}

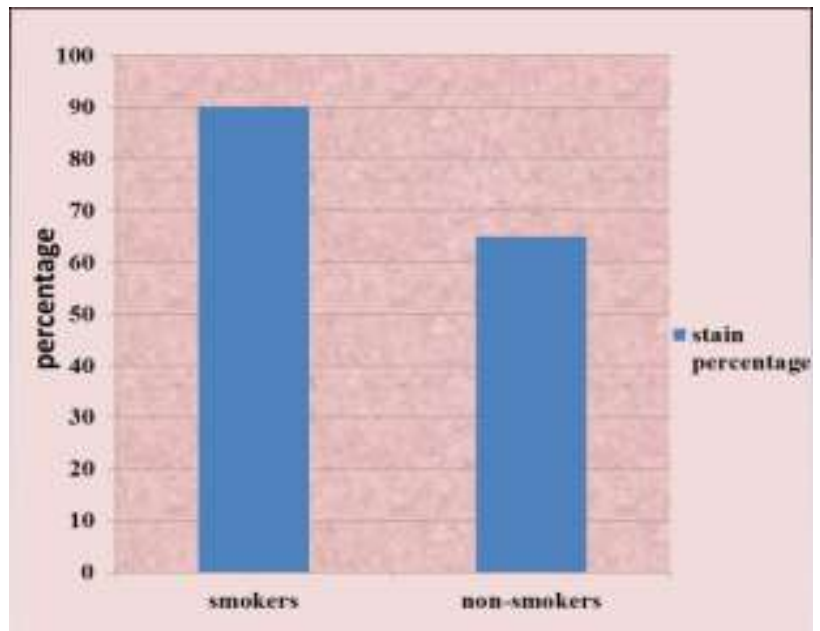

Figure 1: Percentage of dental stain among smokers and non-smokers

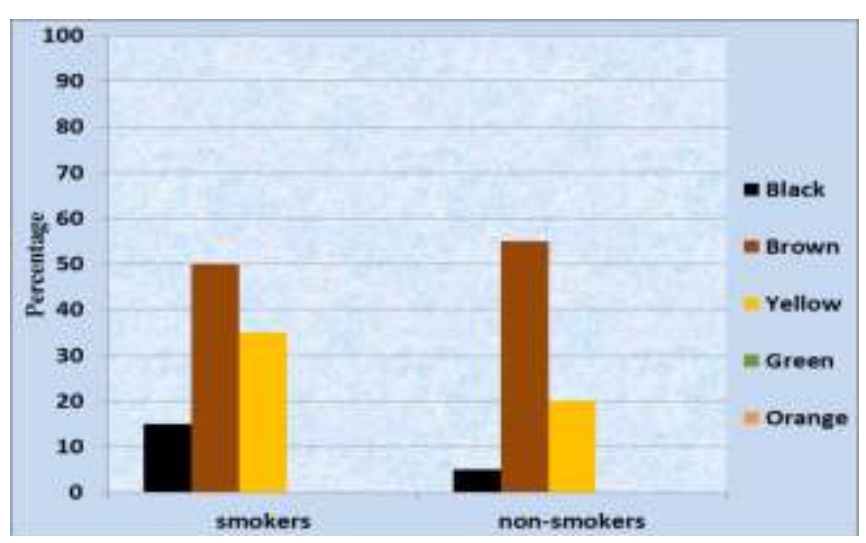

Figure 2: Distribution of smokers and non-smokers according to type of dental stain

\section{Discussion}

The relationship between extrinsic tooth stain and smoking is well known to dentist and it is well established by result of this study which shows that not all smokers have extrinsic tooth stain on their teeth, while finding of Abd-alhameed (1989) [9] showed that all smokers would have some kind of extrinsic tooth stain on their teeth.

According to the result of this study; smoking produce brown, yellow or black stain on the smoker's teeth, but the brown stain is the most extrinsic tooth stain present on the teeth of smokers, findings of Abd-alhameed (1989) [9] showed that there is appositive correlation between smoking and brown stain.
During the study it's been found that there are subjects from smokers group had no dental stain in spite of the long duration of smoking and the cause could be the good oral hygiene and the dental awareness they had. And according to the results of this study the stain became darker with the increase of years of smoking that no yellow stain found on 9-12 years of smoking while increase in number of subjects with the brown stain.

This study show that maximum type of stain when the number of cigarette smoking is from 41 to 80 cigarettes is brown stain and that could relate to the well education of the subjects.

I recommend that people not smoke and smokers should stop smoking because smoking cause many harms, not only on the general and oral health but also the bad appearance of the teeth and gingiva that occur due to smoking.

\section{Conclusion}

1. Both smokers and non-smokers may have some kind of extrinsic tooth stain on their teeth and may not have any stain.

2. Smoking not necessarily the cause of tooth stain.

3. Brown stain is the most type of extrinsic tooth stain found on teeth of both smokers and non-smokers.

4. Black stain is the less type of extrinsic tooth stain found on teeth of both smokers and non-smokers.

\section{References}

[1] Brodeur JM, Payette M, Benigeri M, Charbonneau A, Olivier M, Chabot D. Periodontal diseases among Quebec adults aged 35 to 44 years. J Can Dent Assoc 2001, 67(1):34

[2] Bates C. Jarvis, M \& Connelly, Tobacco additives: cigarette engineering and nicotine addiction, Action on Smoking and Health, Imperial Cancer Research Fund and Massachusetts Tobacco Control Program, November 2006

[3] WHO - World Health Organization 'Tobacco smoke and involuntary smoking', Monograph on the evaluation of carcinogenic risks to humans, WHO International Agency for Research on Cancer, Lyon, France, 2004, Vol. 83.

[4] Caumo W. et al, "Risk factors for preoperative anxiety in adults" Acta Anaestheiologica Scandinavica, 2001, 45(3):298-307..

[5] DeSanto from Georgetown Hospital's community pediatrics program Campaign for Tobacco-Free Kids, September 17, 2009

[6] Haber Wattles, J. Crowley, M. Mandeli, R.Joshipura K. and Kent R L. Evidence for Cigarette Smoking as a Major Risk Fac-tor for Periodontitis, J. Periodontal, 64, 16. January, 1993

[7] SruthyPrathap, H. Rajesh, Vinitha. A. Boloor and Anupama. S. Rao Dept. of Periodontics, Yenepoya Dental College, Nithyananda Nagar Post, Deralakatte, Mangalore575018, Karnataka, India. J. Acad. Indus. Res, 2013; 1(8).

[8] Esthe M. Wilkins. Clinical practice of the dental hygienist 9th edition, 2005.

[9] Abd-Alhameed A. K. extrinsic tooth stains among Iraqi children and adolescents. A master thesis, prevention department, college of dentistry university of Bagdad, 1989 